\section{Salt and Hypertension}

\author{
CESAR ROMERO
}

Renal Division, School of Medicine,

Emory University, Atlanta,

Georgia, USA.

DOI:10.30824/2106-7

A high-salt intake is a pernicious habit, especially for people with hypertension, heart, and kidney diseases. For many years, epidemiological studies have shown negative outcomes of high salt intake. More recently, scientific evidence is elucidating the pathophysiological mechanisms associated with this habit. Some of these concepts have been presented at the $2021 \mathrm{ESH}-\mathrm{ISH}$ Joint Meeting. The classical view that high-salt diet increases fluid intake, inducing extracellular space expansion, to later eliminate excess sodium and water has been challenged recently. A new mechanism, named natriuretic-ureotelic regulation, has been proposed, where the kidneys optimize the renal concentration mechanisms to excrete more sodium and less free water, preventing sodiumassociated osmotic diuresis ${ }^{1}$. This requires the generation of a massive production of osmolytes (urea) from a catabolic-proteolytic state (mainly in skeletal muscle). The subsequent muscle and liver urea generation ultimately increases plasma urea concentration, expands extracellular space, potentiates the kidney medullary concentration mechanism with less free water excretion, and favors metabolic water generation. Additionally, the muscle's catabolic state is accompanied by a significant energy expenditure of the osmolyte generation. This process has been demonstrated by administering high-salt diets to rodents and a few healthy humans but not in larger population studies. However, during the Glasgow meeting, Giacomo Rossito (Glasgow, UK) presented data exploring the natriuretic-ureotelic regulation hypothesis in a retrospective cohort analysis of essential hypertensive patients. Using metabolomic analysis, he showed that patients with high salt intakes (> $5 \mathrm{gr} /$ day) presented higher urinary sodium excretion with less free water excretion, higher urea levels, and more end products of protein catabolism or the urea cycle than those with low salt intakes. These results support the smaller original studies, proposing

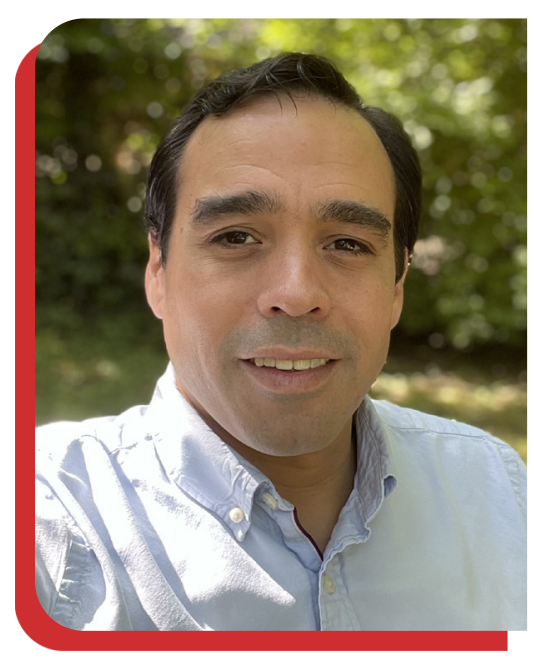

a new concept in salt and water homeostasis. It is unclear how much the natriuretic-ureotelic mechanism affects hypertension genesis and hypertensive organ damage. However, this study obligates physicians and researchers to consider this mechanism in the study of salt and water homeostasis. Additionally, the Rossito's research has confirmed that patients on high-salt diets exhibit higher rates of glomerular filtration and glomerular hyperfiltration. Previous studies in animals have shown that a high salt intake could lead to glomerular hyperfiltration. These results may arise from a positive autoregulatory feedback mechanism on the connecting tubule, mediated by the epithelial sodium channel $(\mathrm{ENaC})$, called connecting tubule-glomerular feedback, that may explain these findings ${ }^{2}$.

During the ESH-ISH Meeting, chloride has also been discussed, as problems related to a highsalt diet involve not only sodium but also chloride. Thus, previous studies have shown that replacing chloride with other anions, such as citrate, evokes neither the same extracellular space expansion nor the blood pressure changes associated with high-salt diets. Additionally, specific chloride transports, such as the bicarbonate-chloride exchanger Pendrin, are key in sodium reabsorption and blood pressure regulation. In this regard, Dr. Michael Stowasser (Brisbane, Australia) explored the expression of kidney transports through urinary extracellular vesicles (UEVs) analysis coupled with liquid chromatography tandem mass spectrometry. Their results showed for the first time in humans the increased abundance of Pendrin in patients with primary aldosteronism compared with low-renin essential hypertensive patients. However, no changes in the sodiumchloride channel (NCC) or aquaporin 2 (Aqp 2) were observed. Unfortunately, no ENaC subunits were detected in the uEVs. These findings also confirm previous studies in rodents, where 
Pendrin abundance was observed in aldosteroneinfused animals. Additionally, chloride's role was highlighted during an oral kidney session at the conference. Dr. Puyol (Buenos Aires, Argentina) presented the effect of the anion chloride on the induction of oxidative stress on a high-salt diet. This revealed the chloride anion was associated with more glutathione peroxidase activity than the sodium cation in the kidney cortex, favoring a prooxidative state.

Outside of the kidney, Dr. Constantino ladecola (NY, USA) revealed the mechanisms associated with high salt intake and cognitive impairment. In animal studies, high salt, independently of blood pressure levels, induces endothelial dysfunction in the brain. This process is mediated by II-17 generated in $\mathrm{TH} 17$ lymphocytes at the intestine, highlighting the role of the gut-brain axis. Thus, II-17 in cerebral arteries decreases endothelial nitric oxide synthase (eNOS), decreasing cerebral blood flow but also increasing accumulation of the phosphorylated Tau protein. These elegant studies showed that the salt-induced Tau protein, induced by high salt, and not decreased blood flow, induced the cognitive impartment. Thus, future clinical trials should translate these findings to prevent cognitive impairment in patients.

Overall, the ESH-ISH Meeting has contributed new concepts and translational evidence on the role of a high-salt diet in hypertension and hypertensionmediated organ damage.

\section{References:}

1.Rakova N, Kitada K, Lerchl K, Dahlmann A, Birukov A, Daub S, et al. Increased salt consumption induces body water conservation and decreases fluid intake. J Clin Invest. 2017;127(5):1932-43. DOl: 10.1172/ $\mathrm{JCl} 88530$

2.Romero CA, Carretero OA. A Novel Mechanism of Renal Microcirculation Regulation: Connecting Tubule-Glomerular Feedback. Curr Hypertens Rep. 2019;21(1):8. DOI: 10.1007/s11906-019-0911-5

\section{Salt and Hypertension - Clinical Aspects}

\section{NEUSA JESSEN \\ Unidade de Investigação, Departamento de Medicina do Hospital Central de Maputo, Maputo, Moçambique.}

DOI:10.30824/2106-8

The clinical importance of high blood pressure has been recognized for almost two centuries now ${ }^{1}$ but its etiopathogenesis is still a matter of continuing intense research. Although effective treatment is available, control of blood pressure (BP) remains a global challenge and hypertension continues to be the main risk factor for morbidity and mortality around the world ${ }^{2}$. The simplified and concise 2020 International Society of Hypertension Global Hypertension Practice Guidelines will harmonize the management for worldwide healthcare providers ${ }^{3}$ while the search for more effective ways of preventing and controlling hypertension continues. It is well stablished that the onset of hypertension may be prevented, or at least delayed, by healthy lifestyle choices ${ }^{4}$, which is also the first line of antihypertensive treatment.

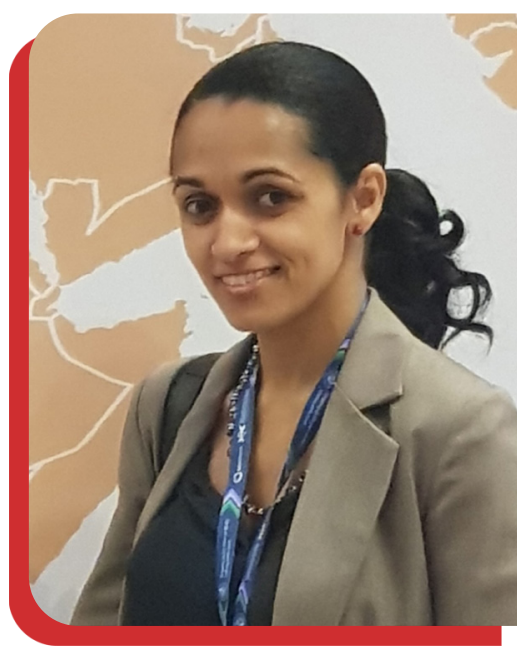

Salt intake is considered the most important dietary risk factor for hypertension, and, in fact, a large body of evidence supports the strong association between high $\mathrm{Na}$ intake and raised $\mathrm{BP}^{5}$. Nevertheless, the susceptibility to the BP-raising effects of salt, called salt sensitivity of BP (SSBP), is a physiological quantitative trait that varies between individuals and the mechanisms that mediate the pressor effects of salt are not fully explained ${ }^{6}$. Furthermore, although the associations between high sodium intake and increased BP as well as between hypertension and cardiovascular (CV) morbidity and mortality are well stablished and undisputed, the question of impact of dietary salt reduction in hard CV outcomes (stroke, myocardial infarction or death) remains unanswered, with several studies presenting opposed results ${ }^{5}$. As 